\title{
Systematic Review of Post Covid Myalgia Literature
}

\section{Manjiri Ranade*, Prakash Deshmukh, Sweety Purushottham and Prasad Kasliwal}

Manjiri Ranade*, Prakash Deshmukh, Sweety Purushottham and Prasad Kasliwal

*Corresponding Author: Manjiri Ranade, Interventional Pain Physician, Palash Pain and Palliative Care, India.
Received: December 16, 2020

Published: January 28, 2021

(C) All rights are reserved by Manjiri Ranade., et al.

\section{Abstract}

Since the end of 2019, the whole world has been suffering through the pandemic of the new Severe Acute Respiratory Syndrome Coronavirus (SARS-CoV-2). Pain is a most common symptom during Novel Coronavirus Disease 2019 (COVID-19). According to the World Health Organization, patients suffering from COVID-19 show symptoms of muscle pain (myalgia) and/or joint pain (arthralgia), sore throat and headache. We present a narrative review of musculoskeletal manifestation and available treatment options. Our aim is to update the pain physicians and physicians working with COVID-19 Patients about possible pathogenesis of myalgia and best proposed treatment available.

Research data for this article is based on literature search which has been referenced in the text. There was no preliminary data. A search of PubMed and Google Scholar was done. We found 15 scientific papers on the related topics. Based on the review we infer that in addition to the cytokine storm experienced by many COVID-19 patients, certain additional factors such as severity of COVID-19 infection contributes to post covid myalgia and fatigue.

Keywords: Coronavirus; Neuropathy; Myalgia; Arthralgia; Headache; Opioids

\section{Introduction}

The aim of this article is to collect currently available international literature on pathophysiology of covid myalgia and available treatment options.

\section{Methods}

A systematic literature search and critical review of collected studies were conducted. An electronic search was done with the help of search engines PubMed and Google Scholar. Search terms used were COVID-19, SARS-CoV-2, Myalgia and Post Viral Fatigue. Identified articles were read and cross referenced and relevant literature was identified. Study selection and extraction of data was used as data collection process. This was done by two investigators and verified by other two. The consensus was made. This study did not involve any living objects hence was exempt from institutional review board approval. Research papers published in English language were used as a reference.

\section{Discussion}

Patients suffering from viral infections including COVID-19 show myalgia as a common symptom. Myalgia shows generalized inflammation and cytokine response. This can be a primary symptom of about $36 \%$ of patients with COVID-19 [1]. Patients suffering from COVID-19 may exacerbate myalgia and fatigue for prolonged duration as compared to patients with other viral infections. They may be unresponsive to conventional painkillers.

In addition to classic mechanism of myalgia known in viral fever the mechanism of musculo skeletal pain could be completely dif- 
ferent in COVID-19. At low cytosolic pH, COVID-19 can enter the cell by penetrating ACE2 and can cause infection in the pulmonary system [2,3]. As ACE2 receptors presence is also there the brain tissue, kidney and musculoskeletal system these organs are also at a risk $[4,5]$. It has been reported in a recent article that surplus cell damage during COVId-19 infection causes increase in lactate levels thereby causing hyperlactemia [6]. Due to hyperlactemia, erythrocytes lose oxygen carrying capacity to the tissues and the thus tissues remain hypoxic.

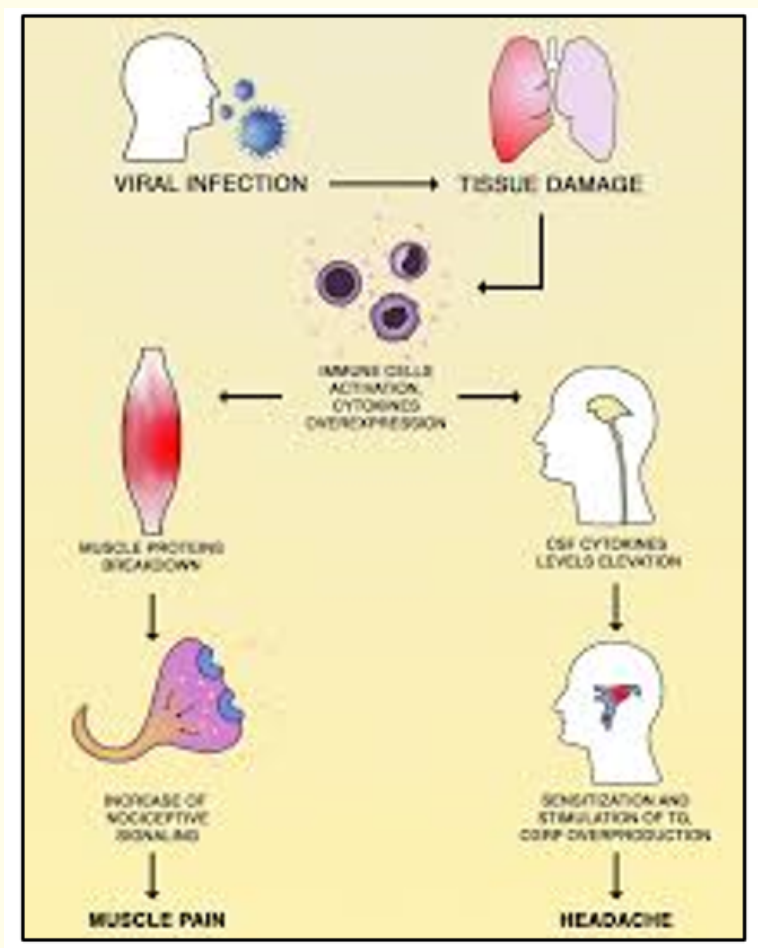

Figure 1: Mechanisms of Myalgia during viral infection.

The virus can infect all tissues containing ACE2 such as the heart, brain, kidney and also musculoskeletal system as it can spread through the bloodstream or vascular endothelium. Muscle involvement during COVID-19 infection is proven by increasing creatinine kinase levels.

Lactate begins to accumulate in the muscles, when muscle tissue cannot provide the necessary energy in aerobic ways during exercise. To prevent lactate accumulation there is an activation of Monocarboxylate transporter (MCT) (synonym, lactate/H+ ion symporter) [7]. MCT pumps lactate and $\mathrm{H}+$ ion, both from the extracellular space to the intracellular space and also to the mitochondria. Lactate dehydrogenase (LDH) increases the lactate formation from pyruvate in anaerobic condition [7,8]. During exercise MCTs will increase between 32 and 76\% and MCT capacities may increase 3 to 4 times during extensive exercise $[7,8]$. Cytosol pH decreases due to accumulation of lactate and $\mathrm{H}+$ ion. This is due to exceeding the capacity of MCTs and other regulatory pumps [8]. Anaerobic glycolysis reduces ATP synthesis. As an end result pain and fatigue are due to decreased ATP synthesis and low intracellular $\mathrm{pH}[7,9]$.

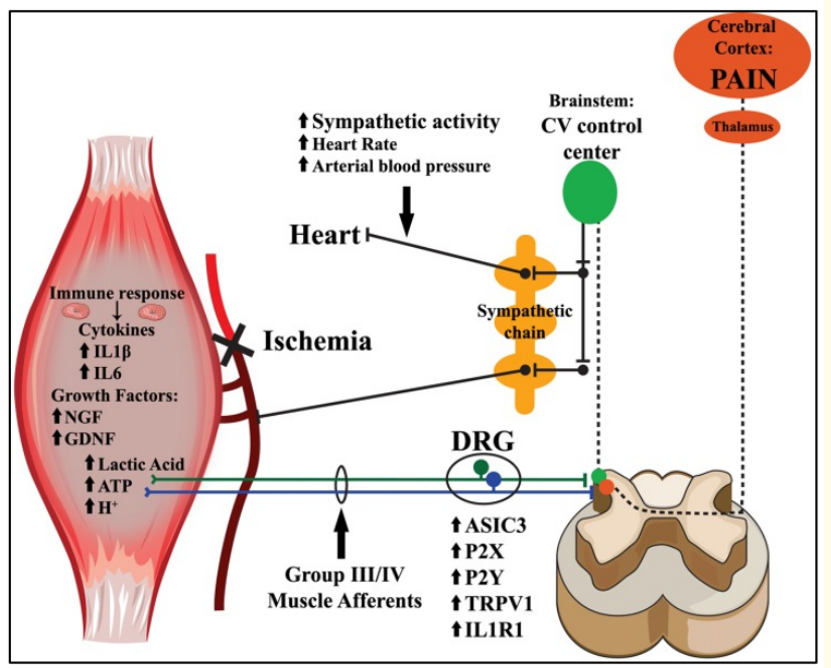

Figure 2: Peripheral Mechanisms of Ischemic Myalgia.

Musculoskeletal system remains deoxygenated due to lack of oxygen transport to the tissue. This is due to erythrocytes losing the oxygen carrying capacity in hyperlactatemia Muscles may show ischemic changes during COVID-19 infection. There is an increase of growth factors and cytokine levels during hypoxic ischemia. Ischemic conditions, and microvascular changes can cause overexpression in the dorsal root ganglion triggering pain [10]. As the virus damages muscles and other tissues LDH levels will increase. This coupled with anaerobic glycolysis increases lactate level excessively.

Post COVID-19 Syndrome, a long term state of chronic fatigue is experienced by some of the COVID-19 affected patients. This is 
described by post-exertional neuroimmune exhaustion [11]. Interlukin 6 and Interlukin 10 are predictors of development of chronic fatigue as they are known to promote inflammatory changes in the body.

SAR's and MER's show similar long term respiratory musculoskeletal and neuro, psychiatric sequeale. These other coronaviruses also have similar pathophysiological findings to COVID-19.

Symptoms

- Persistent fatigue

- Diffused myalgia

- Depressive symptoms

- Non restorative sleep.

In post COVID myalgia symptoms of three most common muscular disorders such as Chronic Fatigue Syndrome, Polymyalgia Rheumatica and Fibromyalgia may exacerbate. In many cases arthralgia is also associated with myalgia.

\section{Treatment}

Presently very few clinical trials or specific guidelines are present for managing pain in COVID-19 patients [12,13]. The aim of this review is to discuss the problems associated with pain management during the COVID-19 pandemic.

\section{NSAIDs and non-opioid analgesics}

Nonsteroidal Anti-Inflammatory Drugs (NSAIDs) are most commonly used drugs for managing pain. They are very popular due to easy accessibility and efficacy as anti-inflammatory and analgesic agents. There are higher chances of exacerbation of respiratory tract infections in COVID-19 patients after use of NSAID's [14]. Also NSAID's are known to have higher side effects. The WHO evaluated 73 studies in which adults and children treated with NSAIDs for respiratory tract infection but none of the studies showed the infection was caused by COVID-19, SARS or MERS [15]. There is no contraindication for using NSAID's. SARS-CoV-2 infections are constantly increasing worldwide. Mild and moderate clinical symptoms of COVID-19 patients are treated mainly with the supportive therapy. Overall, discontinuing the use of NSAID's for pain management in patients with suspected or diagnosed SARS-CoV2 infection is not recommended by EMA (European Medicines Agency) and WHO [16].

\section{Opioids}

Use of Opioids during COVID-19 pandemic for treating pain cannot be justified unambiguously as there are no clinical trials conducted. In patients with severe pulmonary disease Opioids have been found to increase both mortality and the risk of adverse clinical events [17-19]. Opioids is also known to cause immunosuppression. In an animal model (Flores., et al.) found that morphine induces adrenal-dependent lymphopenia and reduces the response to mitogenic stimulation (dose $=10 \mathrm{mg} / \mathrm{kg}$ ) by nearly $70 \%[20,21]$.

Tramadol and buprenorphine are superior that other Opioid's. Both of them do not have any immunosuppressive properties, so, theoretically, they do not prolong viral shedding [22,23]. Buprenorphine is safe in multi-organ failure and it has a ceiling effect for respiratory depression $[22,23]$.

\section{Corticosteroids}

Systemic corticosteroids have been shown to inhibit interferon (ifn) pathway of innate immunity. Some reports published recently also states that it may exacerbate symptoms of COVID-19 while others conflicting ones shows improvement in patients' symptoms. [24]. Based on these findings, WHO recommends cautious corticosteroid use for the pain management [25].

\section{Neuropathic pain treatment in SARS-CoV-2 infection}

Peripheral nervous system involvement, including painful neuropathies, was reported in many patients with SARS-CoV-1 and now with SARS-CoV-2 infection [26-29]. This may be a consequence of either viral invasion of the peripheral nerves (neurotropism) [30] or prolonged immobility during severe illness [31], or both. Gabapentin and pregabalin are commonly used in the treatment neuropathic pain. While the numbers needed to treat (NNT) for 50\% pain relief for these therapeutic agents are similar (7.2-gabapentin; 7.7-pregabalin) [32,33] pregabalin acts quicker than gabapentin [34]. They are usually well tolerated and characterized by similar adverse effects [32-34]. Their potency in treating neuropathic pain in SARS-CoV2 patients cannot be predicted as no clinical sufficient clinical trials have been done.

\section{Preventive measures}

- Regular gentle stretching exercises

- Deep breathing exercises 
- Small walks in house

- Positive optimistic outlook to reduce stress

- Endurance exercises involving light weights

- Hot fermentation and gentle massage for tight muscles

- Drinking lot of water and healthy well balanced diet.

- Regular change of posture and avoiding sitting in one position for long period.

\section{Conclusion}

Pain symptoms caused by the virus includes myalgia, arthralgia, sore throat, headache and peripheral neuropathies. For the treatment of pain, each patient requires an individual approach based on available knowledge and, more importantly, the patient's condition and comorbidities. The information provided is a cross-section of the available knowledge aimed at improving the patient's clinical condition. A structured prospective evaluation should be undertaken to analyse the probability, severity, sources and adequate treatment of pain in patients with COVID-19 infection and those suffering due to an unavailability of pain services during the COVID-19 pandemic.

\section{Bibliography}

1. Lippi G., et al. "Myalgia may not be associated with severity of coronavirus disease 2019 (COVID-19)". World Journal of Emergency Medicine 11 (2020): 193-194.

2. Cure E and Cumhur Cure M. "Comment on "Organ-protective Effect of Angiotensin-converting Enzyme 2 and its Effect on the Prognosis of COVID-19". Journal of Medical Virology (2020).

3. Cure E and Cumhur Cure M. "Angiotensin-converting enzyme inhibitors and angiotensin receptor blockers may be harmful in patients with diabetes during COVID-19 pandemic". Diabetes and Metabolic Syndrome 14 (2020): 349-350.

4. Cure E and Cumhur Cure M. "Comment on 'Should COVID-19 Concern Nephrologists? Why and to What Extent? The Emerging Impasse of Angiotensin Blockade". Nephron 144 (2020): 251-252.

5. Echeverría-Rodríguez 0., et al. "Angiotensin- (1-7) participates in enhanced skeletal muscle insulin sensitivity after a bout of exercise". Journal of the Endocrine Society 4 (2020).
6. Cure E and Cumhur Cure M. "Can dapagliflozin have a protective effect against COVID-19 infection? A hypothesis". Diabetes and Metabolic Syndrome 14 (2020): 405-406.

7. Juel C and Halestrap AP. "Lactate transport in skeletal muscle role and regulation of the monocarboxylate transporter". Journal of Physiology 517 (1999): 633-642.

8. Messonnier L., et al. "Importance of $\mathrm{pH}$ regulation and lactate/ $\mathrm{H}+$ transport capacity for work production during supramaximal exercise in humans". Journal of Applied Physiology 102 (2007): 1936-1944.

9. Liang CZ., et al. "The relationship between low $\mathrm{pH}$ in intervertebral discs and low back pain: a systematic review". Archives of Medical Science 8 (2012): 952-956.

10. Queme LF, et al. "Peripheral mechanisms of ischemic myalgia". Frontiers in Cellular Neuroscience 11 (2017): 419.

11. https://scroll.in/article/967801/why do some coronavirus patients suffer chronic fatigue even after recovery

12. Madhok J and Mihm FG. "Rethinking sedation during prolonged mechanical ventilation for COVID-19 respiratory failure". Anesthesia and Analgesia 131 (2020): e123-e124.

13. Hanidziar D and Bittner E. "Sedation of mechanically ventilated COVID-19 patients: Challenges and special considerations". Anesthesia and Analgesia 131 (2020): e40-e41.

14. Russell B., et al. "COVID-19 and treatment with NSAIDs and corticosteroids: Should we be limiting their use in the clinical setting?" Ecancermedicalscience 14 (2020): 1023.

15. World Health Organization. The Use of Non-Steroidal AntiInflammatory Drugs (NSAIDs) in Patients with COVID-19 (2020).

16. Shanthanna H., et al. "Caring for patients with pain during the COVID-19 pandemic: Consensus recommendations from an international expert panel”. Anaesthesia 75 (2020): 935-944.

17. Vozoris NT. "Opioid utility for dyspnea in chronic obstructive pulmonary disease: A Complicated and controversial story". Annals of Palliative Medicine 9 (2020): 571-578. 
18. Vozoris NT., et al. "Predictors of opioid-related adverse pulmonary events among older adults with COPD". Annals of the American Thoracic Society (2020).

19. Vozoris NT., et al. "Incident opioid drug use and adverse respiratory outcomes among older adults with COPD". European Respiratory Journal 48 (2016): 683-693.

20. Flores LR., et al. "Mechanisms of morphine-induced immunosuppression: Effect of acute morphine administration on lymphocyte trafficking". Journal of Pharmacology and Experimental Therapeutics 272 (1995): 1246-1251.

21. Hernandez MC., et al. "Immunosuppression by Morphine is mediated by central pathways". Journal of Pharmacology and Experimental Therapeutics 267 (1993): 1336-1341.

22. Davis MP. "Twelve reasons for considering buprenorphine as a frontline analgesic in the management of pain". Journal of Supportive Oncology 10 (2012): 209-219.

23. Davis MP., et al. "Treating chronic pain: An Overview of clinical studies centered on the buprenorphine option". Drugs 78 (2018): 1211-1228.

24. Arabi YM., et al. "Corticosteroid therapy for critically Ill patients with middle east respiratory syndrome". American Journal of Respiratory and Critical Care Medicine 197 (2018): 757767.

25. WHO. Clinical Management of Severe Acute Respiratory Infection When Novel Coronavirus (NCoV) Infection Is Suspected; World Health Organization: Geneva, Switzerland (2020).

26. Mao L., et al. "Neurologic manifestations of hospitalized patients with coronavirus disease 2019 in Wuhan, China". JAMA Neurology 77 (2020): 1-9.

27. Abdelnour L., et al. "COVID 19 infection presenting as motor peripheral neuropathy". Journal of the Formosan Medical Association 119 (2020): 1119-1120.

28. Montalvan V., et al. "Neurological manifestations of COVID-19 and other coronavirus infections: A systematic review". Clinical Neurology and Neurosurgery 194 (2020): 105921.

29. Pleasure SJ., et al. "The spectrum of neurologic disease in the severe acute respiratory syndrome coronavirus 2 pandemic infection: Neurologists move to the frontlines". JAMA Neurology 77 (2020): 679-680.

30. Li YC., et al. "The neuroinvasive potential of SARS-CoV2 may play a role in the respiratory failure of COVID-19 patients". Journal of Medical Virology 92 (2020): 552-555.

31. Devlin JW., et al. "Strategies to optimize ICU liberation (A to F) bundle performance in critically Ill adults with coronavirus disease 2019". Critical Care Explorations 2 (2020): e0139.

32. Finnerup NB., et al. "Pharmacotherapy for neuropathic pain in adults: A systematic review and meta-analysis". Lancet Neurology 14 (2015): 162-173.

33. Brooks KG and Kessler TL. "Treatments for neuropathic pain". Clinical pharmacology 9 (2017): 1-14.

34. Pop-Busui R., et al. "Diabetic neuropathy: A position statement by the american diabetes association". Diabetes Care 40 (2017): 136-154.

\section{Assets from publication with us}

- Prompt Acknowledgement after receiving the article

- Thorough Double blinded peer review

- Rapid Publication

- Issue of Publication Certificate

- High visibility of your Published work

Website: www.actascientific.com/

Submit Article: www.actascientific.com/submission.php Email us: editor@actascientific.com

Contact us: +919182824667 